\title{
Multiscale filter-based hyperspectral image classification with PCA and SVM
}

\author{
Guang Yi Chen ${ }^{1}$
}

\begin{abstract}
Hyperspectral imagery can offer images with high spectral resolution and provide a unique ability to distinguish the subtle spectral signatures of different land covers. In this paper, we develop a new algorithm for hyperspectral image classification by using principal component analysis (PCA) and support vector machines (SVM). We use PCA to reduce the dimensionality of an HSI data cube, and then perform spatial convolution with three different filters on the PCA output cube. We feed all three convolved output cubes to SVM to classify every pixel. Finally, we perform fusion on the three output maps to determine the final classification map. We conduct experiments on three widely used hyperspectral image data cubes (ie indian pines, pavia university, and salinas). Our method can improve the classification accuracy significantly when compared to several existing methods. Our novel method is relatively fast in term of CPU computational time as well.
\end{abstract}

K e y w or d s: hyperspectral image classification; 2 D filter; 2 D convolution; support vector machines (SVM)

\section{Introduction}

Hyperspectral image (HSI) classification is an immensely popular topic in recent years. It has been widely used in agriculture, mineralogy, physics, astronomy, chemical imaging, and environmental sciences. We briefly review some existing techniques for HSI classification here. Guo et al [1] adopted two fusion methods for spectral and spatial features. To improve classification accuracy, they utilized a guided filter to achieve better results and they used support vector machine (SVM) to classify the HSI. Zhou et al [2] developed an HSI method by using spectralspatial long shortterm memory networks. Chen et al [3] proposed a new nonlinear approach for HSI classification. For each pixel in the feature space, a sparse representation vector was chosen by decomposing the pixel over a training dictionary with a kernel-based greedy pursuit method. Melgani and Bruzzone [4] investigated the problem of classifying HSI images by means of SVMs. Fauvel et al [5] studied the classification of HSI images with extremely high spatial resolution. Their approach investigated both the spatial and the spectral information in the remote-sensing images. Camps-Valls and Bruzzone [6] invented the framework of kernel-based methods for HSI classification. They analyzed the performance of regularized radial basis function neural networks, standard SVMs, kernel Fisher discriminant analysis, and regularized AdaBoost. Li et al [7] proposed a new method for the development of generalized composite kernel machines for HSI classification. They studied a new family of generalized composite kernels, which exhibited great flexibility when combining the spectral and the spatial information contained in HSI data. Chen [8] investigated a new sparsity-based method for the classification of HSI. Their approach was based on the observation that a hyperspectral pixel can be sparsely represented by a linear combination of a few training samples. Li [9] developed a new method for spectral-spatial classification of HSI data. Their proposed technique worked as an engine in the context of which active learning algorithms could exploit both spatial and spectral information at the same time. Kang et al [10] developed a new spectral-spatial classification method based on edge-preserving filtering. Cheng et al [11] developed a robust adaptive loss function to measure the representation loss so that it could greatly relieve the side effects of outlier channels. Chen et al [12] introduced deep learning into hyperspectral data classification. They studied the eligibility of stacked autoencoders by following classical spectral information-based classification. Liu et al [13] studied dimensionality reduction of HSI data based on improved spatialspectral weight manifold embedding. The structural weight matrix was formed from within-class and between-class coefficient representation matrices. These matrices were obtained by using the collaborative representation method.

In this paper, we develop a novel method for hyperspectral image (HSI) classification based on principal component analysis (PCA) [14] and SVM [15]. Our new method benefits from both spatial and spectral information at the same time. We choose PCA to reduce the dimensionality of the input HSI data cube. We perform 2 D spatial convolution [16] to every PCA output channel with three $2 \mathrm{D}$ filters, and we feed three convolved cubes to SVMs to generate three classification maps. We perform fusion on every pixel in the three output maps to generate the final classification map. Experimental results

1Department of Computer Science and Software Engineering, Concordia University, Montreal, QC, Canada H3G 1M8, guangyi_chen@hotmail.com

DOI:10.2478/jee-2021-0006, Print (till 2015) ISSN 1335-3632, On-line ISSN 1339-309X

(C) This is an open access article licensed under the Creative Commons Attribution-NonCommercial-NoDerivs License (http: //creativecommons.org/licenses/by-nc-nd/3.0/). 


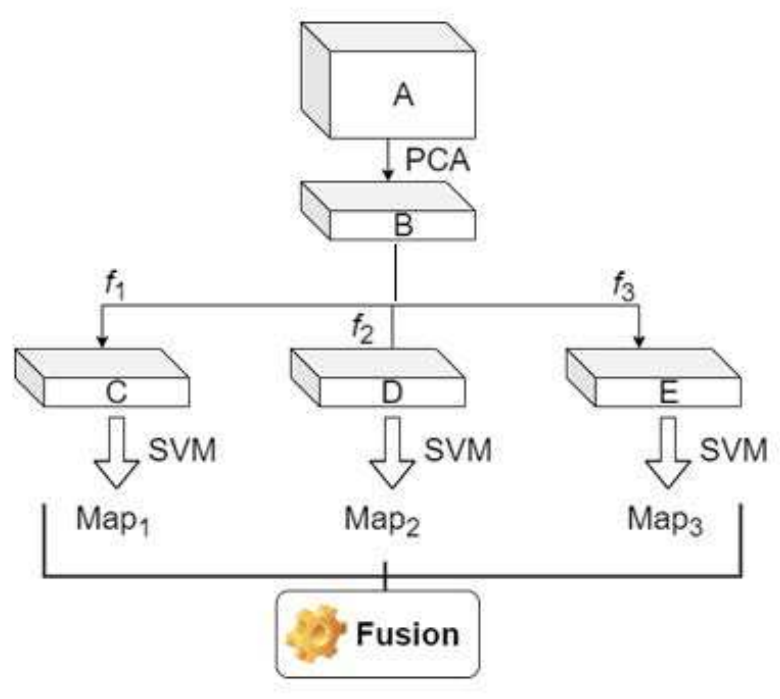

Fig. 1. The flowchart of our new method

show that our new method outperforms several existing methods significantly for HSI classification.

\section{The proposed method}

HSI classification is an extremely hot research topic in remote sensing in recent years. The precise classification of ground features through HSI is extremely important and has attracted widespread attention. Due to noise and water absorption, existing HSI data cubes need to be preprocessed before conventional methods can be applied. The major challenges for HSI classification are (a) curse of dimensionality and (b) insufficient samples pool during training. For a given set of observations with known class labels, the basic aim of HSI classification is to assign a class label to each pixel in the HSI data cube.

PCA [14] is a method for reducing the dimensionality of a dataset, increasing interpretability, and minimizing information loss. It builds new uncorrelated variables that successively maximize variance. PCA can be conducted in the following steps: (a) Take the whole dataset with $\mathrm{P}$ dimensions. (b) Compute the mean for every dimension of the dataset. (c) Find the covariance matrix of the dataset. (d) Calculate the eigenvectors and the eigenvalues of the covariance matrix. (e) Retain d eigenvectors as the output principal components (PC).

An SVM [15] is a machine learning technique, which can generalize between two different classes if the labelled data is provided in the training set. The major duty of the SVM is to check for the hyperplane that can distinguish between two classes. It uses the kernel trick to transform the data and it finds an optimal boundary between the possible outputs. The SVM performs extremely complex data transformations and it finds out how to separate the data according to the labels.

In this paper, we perform PCA to existing HSI data cube to reduce the dimensionality of the HSI data cubes. As we know that spatial information is also useful for HSI classification, so it is better to invent an algorithm that takes advantages of both spatial information and spectral information. We introduce three intermediate sized 2 D filters by experiments and spatially convolve the PCA output cube with them. We perform classification by means of SVM on the three convolved cubes and conduct fusion on three maps to yield the final classification map. Experiments show that our new method generates extremely good results for HSI classification of three popular HSI data cubes. Figure 1 shows the flowchart of this new method.

Our proposed method for HSI classification can be described as follows:

Input:

- A - An HSI data cube that has a spatial resolution of $M \times N$ pixels with $\mathrm{P}$ spectral bands.

- $\mathrm{d}$ - The number of PCA components to be retained, which is determined by experiments.

Output: The class of each spatial pixel in the HSI data cube A.

Perform PCA to the given hyperspectral imagery data cube $\mathrm{A}$ and retain d output components, denoted as $\mathrm{B}$ $(M \times N \times d)$.

- Set intermediate sized $2 \mathrm{D}$ filer $f_{1}=1 / 36 \times$ ones $\left.(6,6)\right)$, where ones $(6,6)$ is an unit matrix of size $6 \times 6$.

- Set intermediate sized $2 \mathrm{D}$ filer $f_{2}=1 / 49 \times$ ones $\left.(7,7)\right)$, where ones $(7,7)$ is an unit matrix of size $7 \times 7$.

- Set intermediate sized $2 \mathrm{D}$ filer $f_{3}=1 / 64 \times$ ones $\left.(8,8)\right)$, where ones $(8,8)$ is an unit matrix of size $8 \times 8$.

- Spatially convolve $B$ with filter $f_{1}$, denoted as $C(M \times N \times d)$.

- Spatially convolve $B$ with filter $f_{2}$, denoted as $D(M \times N \times d)$.

- Spatially convolve $B$ with filter $f_{3}$, denoted as $E(M \times N \times d)$.

- Classify each hyperspectral pixel vector with size $d \times 1$ in $C$ as one of the known classes by SVM classifier. Let the output be $M a p_{1}$.

- Classify each hyperspectral pixel vector with size $d \times 1$ in $D$ as one of the known classes by SVM classifier. Let the output be $\mathrm{Map}_{2}$.

- Classify each hyperspectral pixel vector with size $d \times 1$ in $E$ as one of the known classes by SVM classifier. Let the output be $M a p_{3}$.

- Fuse on $M a p_{1}, M a p_{2}$ and $M a p_{3}$ to generate final map Map.

The major contribution of this paper is as follows. Our novel method utilizes the PCA transform to reduce the dimensionality of HSI data cubes. It performs spatial $2 \mathrm{D}$ convolution three times to the PCA output cube. We select to use the SVM to classify each pixel in the PCA output cube to one of the known classes. We conduct fusion on the three maps generated by SVM to produce the final classification map. Experiments demonstrate that our novel method yields extremely good results for HSI classification of three popular HSI data cubes. Because our novel method only performs simple operations on HSI 


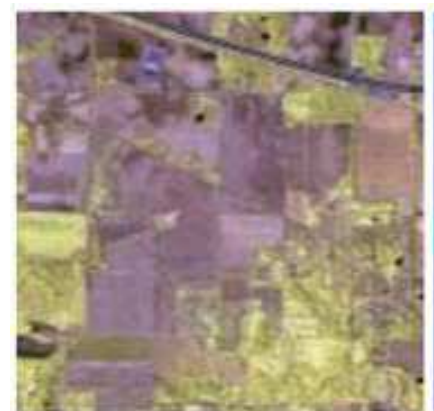

(a)

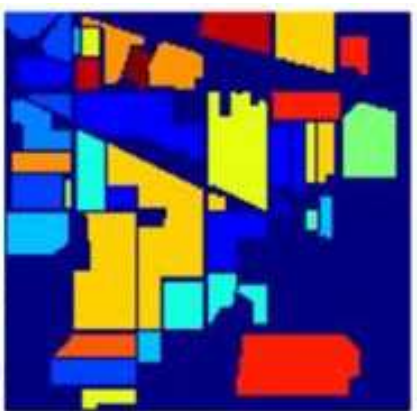

(b)
Fig. 2. Indian pines data cube: (a) - false color composite image, (b) - round truth map with classes (a)

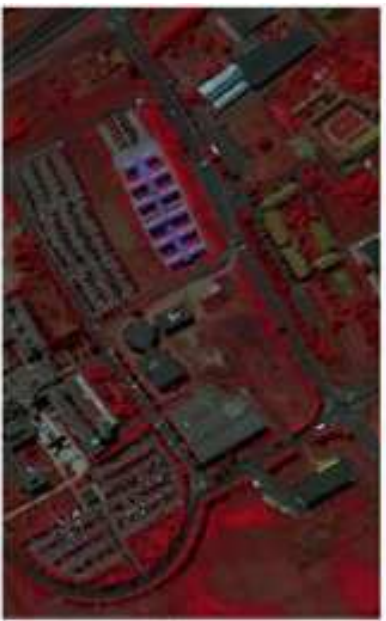

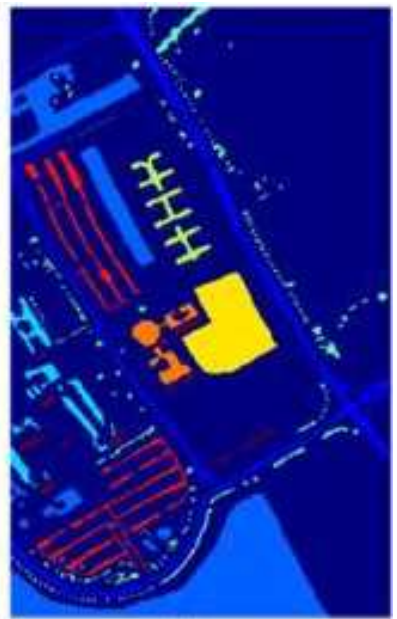

(b)
Fig. 3. Pavia university data cube: (a) - false color composite image, (b) - ground truth map with classes

Table 1. Ground truth classes and the total pixel number for each class in indian pines data cube

\begin{tabular}{lll}
\hline No & Class & $\begin{array}{l}\text { Total } \\
\text { samples }\end{array}$ \\
\hline C1 & Alfalfa & 46 \\
C2 & Corn notill & 1428 \\
C3 & Corn mintill & 830 \\
C4 & Corn & 237 \\
C5 & Grass pasture & 483 \\
C6 & Grass trees & 730 \\
C7 & Grass pasture mowed & 28 \\
C8 & Hay windrowed & 478 \\
C9 & Oats & 20 \\
C10 & Soybean notill & 972 \\
C11 & Soybean mintill & 2455 \\
C12 & Soybean clean & 593 \\
C13 & Wheat & 205 \\
C14 & Woods & 1265 \\
C15 & Buildings Grass Trees Drives & 386 \\
C16 & Stone Steel Towers & 93 \\
\hline
\end{tabular}

data cubes, it is relatively fast in term of CPU computation time.

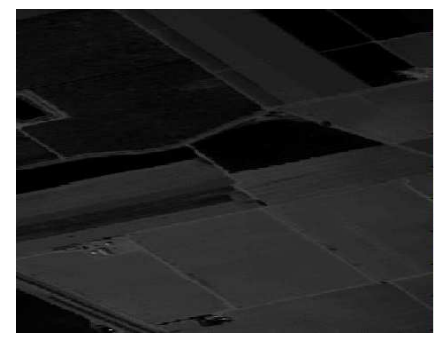

(a)

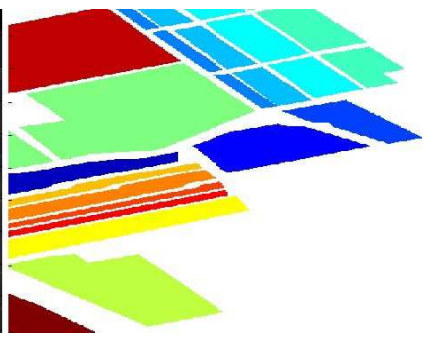

(b)
Fig. 4. Salinas data cube (a) - false color composite image, (b) - ground truth map with classes

\section{Data sites}

The details about the three HSI data cubes that we experimented with are described as follows.

a. Indian Pines. This data cube was acquired by the AVIRIS sensor over the Indian Pine test site in northwestern Indiana, USA, on June 12, 1992. This data cube has $145 \times 145$ pixels with 200 spectral bands. Figure 2 depicts the false-colour composite image and the groundtruth map. Table 1 tabulates ground truth classes and the pixel number for every class in this data cube.

b. Pavia University. This data cube was acquired by the ROSIS sensor during a flight campaign over Pavia, northern Italy, on July 8, 2002. This data cube has $610 \times 340$ pixels with 115 spectral bands. Fig. 3 depicts a 3-band false-colour composite image and the ground truth map. There are 9 classes of land covers, which is tabulated in Table 2.

Table 2. Ground truth classes and the total pixel number for each class in pavia university data cube

\begin{tabular}{lcc}
\hline No & Class Names & Total Samples \\
C1 & Asphalt & 6631 \\
C2 & Meadows & 18649 \\
C3 & Gravel & 2099 \\
C4 & Trees & 3064 \\
C5 & Painted metal sheets & 1345 \\
C6 & Bare Soil & 5029 \\
C7 & Bitumen & 1330 \\
C8 & Self-Blocking Bricks & 3682 \\
C9 & Shadows & 947 \\
\hline
\end{tabular}

c. Salinas. This data cube was collected by the AVIRIS sensor over Salinas Valley, California. This data cube has $512 \times 217$ pixels with 204 spectral bands. Figure 4 demonstrates a false-colour composite image and the ground truth map. Table 3 shows ground truth classes and the total pixel numbers for all classes. 
Table 3. Ground truth classes and the total pixel number for each class in salinas data cube

\begin{tabular}{lcc}
\hline No & Class Names & Total Samples \\
C1 & Broccoli green weeds 1 & 2009 \\
C2 & Broccoli green weeds 2 & 3726 \\
C3 & Fallow & 1976 \\
C4 & Fallow rough plow & 1394 \\
C5 & Fallow smooth & 2678 \\
C6 & Stubble & 3959 \\
C7 & Celery & 3579 \\
C8 & Grapes untrained & 11271 \\
C9 & Soil vinyard develop & 6203 \\
C10 & Corn senesced green weeds & 3278 \\
C11 & Lettuce romaine 4 wk & 1068 \\
C12 & Lettuce romaine 5 wk & 1927 \\
C13 & Lettuce romaine 6 wk & 916 \\
C14 & Lettuce romaine 7 wk & 1070 \\
C15 & Vinyard untrained & 7268 \\
C16 & Vinyard vertical trellis & 1807 \\
\hline
\end{tabular}

\section{EXPERIMENTAL RESULTS}

In our experiments, we select to use LIBSVM [17] as a classifier to classify every pixel in HSI data cube. It is an integrated software for support vector classification,
(C-SVC, nu-SVC), regression (epsilon-SVR, nu-SVR) and distribution estimation (one-class SVM). LIBSVM supports multi-class classification as well. We choose radial basis function as the kernel for SVMs. We randomly choose $5 \%$ of HSI pixels as validation data and the rest of pixels as testing data. We choose the best parameter $\mathrm{C}$ in $\mathrm{C}$-SVC and we use this parameter $\mathrm{C}$ to classify pixels in the whole data cube.

We run our Matlab code for 10 times and obtain the mean overall accuracy and STD. We compare our new method with PCA 14], locally linear embedding (LLE) [18], and ISS-WME [13] in our experiments. The overall accuracy and STD are shown in Tables 4-6 for the Indian Pines data cube, the Pavia University data cube, and the Salinas data cube, respectively. Table 4 randomly selects $10 \%, 20 \%, 30 \%, 40 \%$, and $50 \%$ of pixels as training data set and the rest of pixels as testing data set for each class. Table 5 randomly chooses $2 \%, 4 \%, 6 \%$, $8 \%$, and $10 \%$ of pixels as training data set and the rest of pixels as testing data set for every class. Table 6 randomly picks $2 \%, 4 \%, 6 \%, 8 \%$, and $10 \%$ of pixels as training data set and the rest of pixels as testing data set for each class. The individual class accuracies with $(50 \%$, $10 \%, 11 \%$ ) training pixels and the rest as testing pixels are shown in Tables 7-9 for the three HSI data cubes (Indian Pines, Pavia University, Salinas), respectively. We choose $d=30$ output channels by experiments for our PCA transform for all three HSI data cubes. The results

Table 4. Results of different methods for the Indian Pines data set with $d=30$ (Overall Accuracy \pm STD)

\begin{tabular}{cccccc}
\hline Percentage (\%) & RAW & PCA & LLE & ISS-WME & Proposed \\
10 & $49.82 \pm 1.94$ & $68.40 \pm 1.14$ & $65.93 \pm 1.71$ & $75.38 \pm 1.47$ & $96.91 \pm 0.51$ \\
20 & $51.86 \pm 1.59$ & $72.34 \pm 1.47$ & $71.42 \pm 1.27$ & $81.25 \pm 1.51$ & $98.76 \pm 0.23$ \\
30 & $53.11 \pm 1.35$ & $74.82 \pm 1.69$ & $74.37 \pm 1.48$ & $83.83 \pm 1.73$ & $99.27 \pm 0.15$ \\
40 & $54.28 \pm 1.81$ & $76.07 \pm 1.44$ & $75.98 \pm 1.21$ & $84.80 \pm 1.80$ & $99.65 \pm 0.10$ \\
50 & $54.77 \pm 1.39$ & $76.65 \pm 1.76$ & $76.67 \pm 1.27$ & $84.71 \pm 0.93$ & $99.73 \pm 0.06$ \\
\hline
\end{tabular}

Table 5. Results of different methods for the Pavia University data set with $d=30$ (Overall Accuracy \pm STD)

\begin{tabular}{cccccc}
\hline Percentage (\%) & RAW & PCA & LLE & ISS-WME & Proposed \\
2 & $58.42 \pm 1.13$ & $79.71 \pm 1.18$ & $79.05 \pm 1.42$ & $84.17 \pm 0.87$ & $97.34 \pm 0.19$ \\
4 & $59.62 \pm 1.51$ & $82.60 \pm 1.74$ & $82.52 \pm 1.04$ & $85.96 \pm 0.99$ & $98.45 \pm 0.11$ \\
6 & $71.81 \pm 1.27$ & $84.98 \pm 1.84$ & $85.32 \pm 1.32$ & $87.13 \pm 1.49$ & $99.00 \pm 0.05$ \\
8 & $70.14 \pm 1.22$ & $85.52 \pm 0.87$ & $84.56 \pm 1.21$ & $86.91 \pm 1.40$ & $99.20 \pm 0.08$ \\
10 & $70.99 \pm 1.31$ & $85.75 \pm 1.29$ & $75.02 \pm 0.73$ & $86.98 \pm 1.12$ & $99.44 \pm 0.06$ \\
\hline
\end{tabular}

Table 6. Results of different methods for the Salinas data set with $d=30$ (Overall Accuracy \pm STD)

\begin{tabular}{cccccc}
\hline Percentage (\%) & RAW & PCA & LLE & ISS-WME & Proposed \\
2 & $63.34 \pm 1.13$ & $75.12 \pm 2.18$ & $89.23 \pm 2.42$ & $88.53 \pm 1.23$ & $98.84 \pm 0.23$ \\
4 & $66.47 \pm 1.51$ & $77.25 \pm 1.34$ & $89.38 \pm 2.94$ & $90.22 \pm 0.99$ & $99.41 \pm 0.12$ \\
6 & $68.01 \pm 1.27$ & $77.56 \pm 1.84$ & $91.61 \pm 2.32$ & $91.90 \pm 1.29$ & $99.71 \pm 0.08$ \\
8 & $68.96 \pm 2.22$ & $77.96 \pm 1.87$ & $91.92 \pm 3.21$ & $91.16 \pm 1.04$ & $99.83 \pm 0.04$ \\
10 & $69.13 \pm 1.21$ & $79.02 \pm 2.29$ & $92.16 \pm 2.73$ & $92.19 \pm 1.02$ & $99.87 \pm 0.05$ \\
\hline
\end{tabular}


Table 7. Classification results of different methods for every class for the Indian Pines data set (Overall Accuracy) with $d=30$ and $50 \%$ training pixels.

\begin{tabular}{lccccc}
\hline Class Names & RAW & PCA & LLE & ISS-WME & Proposed \\
Alfalfa & 13.04 & 52.17 & 30.77 & 86.96 & 99.56 \\
Corn notill & 38.42 & 69.37 & 40.06 & 72.17 & 99.55 \\
Corn mintill & 25.06 & 48.76 & 44.34 & 56.47 & 99.71 \\
Corn & 14.41 & 77.11 & 26.27 & 53.95 & 99.75 \\
Grass pasture & 59.06 & 90.87 & 62.38 & 94.65 & 99.79 \\
Grass trees & 86.48 & 97.81 & 97.90 & 98.86 & 99.95 \\
Grass pasture mowed & 35.71 & 76.19 & 50.28 & 83.81 & 100 \\
Hay windrowed & 88.70 & 99.86 & 97.13 & 99.68 & 100 \\
Oats & 11,24 & 43.33 & 30.00 & 86.67 & 100 \\
Soybean notill & 25.17 & 63.51 & 94.24 & 75.17 & 99.65 \\
Soybean mintill & 71.15 & 83.32 & 73.62 & 79.52 & 99.74 \\
Soybean clean & 56.41 & 64.75 & 52.70 & 74.07 & 99.36 \\
Wheat & 74.51 & 94.12 & 75.21 & 99.87 & 99.80 \\
Woods & 94.57 & 97.68 & 86.71 & 97.66 & 100 \\
Buildings Grass Trees Drives & 29.02 & 45.77 & 34.20 & 52.85 & 100 \\
Stone Steel Towers & 91.30 & 90.58 & 88.70 & 98.41 & 97.39 \\
Overall Accuracy & 54.77 & 76.65 & 76.67 & 84.71 & 99.73 \\
\hline
\end{tabular}

Table 8. Classification results of different methods for every class for the Pavia University data set (Overall Accuracy) with $d=30$ and $10 \%$ training pixels

\begin{tabular}{lccccc}
\hline Class Names & RAW & PCA & LLE & ISS-WME & Proposed \\
Asphalt & 62.96 & 88.41 & 86.10 & 88.03 & 99.51 \\
Meadows & 91.90 & 97.72 & 96.66 & 97.42 & 99.99 \\
Gravel & 45.72 & 50.71 & 59.04 & 84.23 & 96.32 \\
Trees & 39.96 & 84.75 & 86.34 & 89.65 & 98.50 \\
Painted metal sheets & 98.51 & 99.61 & 99.72 & 100 & 99.98 \\
Bare Soil & 46.54 & 66.36 & 48.25 & 87.44 & 99.97 \\
Bitumen & 45.02 & 49.72 & 65.27 & 80.38 & 99.52 \\
Self-Blocking Bricks & 54.43 & 84.45 & 83.27 & 86.38 & 98.26 \\
Shadows & 46.57 & 99.37 & 99.68 & 100 & 98.79 \\
Overall Accuracy & 70.99 & 85.75 & 75.02 & 86.98 & 99.44 \\
\hline
\end{tabular}

Table 9. Classification results of different methods for every class for the Salinas data set (Overall Accuracy) with $d=30$ and $11 \%$ training pixels

\begin{tabular}{lccccc}
\hline Class Names & RAW & PCA & LLE & ISS-WME & Proposed \\
Broccoli green weeds 1 & 91.26 & 93.14 & 96.68 & 98.01 & 100 \\
Broccoli green weeds 2 & 99.22 & 99.28 & 95.53 & 99.88 & 100 \\
Fallow & 61.75 & 81.33 & 93.59 & 93.36 & 100 \\
Fallow rough plow & 96.49 & 97.29 & 95.37 & 99.20 & 99.58 \\
Fallow smooth & 80.00 & 83.24 & 83.65 & 98.34 & 99.58 \\
Stubble & 95.29 & 96.07 & 86.59 & 99.94 & 99.99 \\
Celery & 97.21 & 89.67 & 88.33 & 99.75 & 99.99 \\
Grapes untrained & 75.75 & 83.28 & 83.59 & 99.48 & 99.81 \\
Soil vinyard develop & 98.64 & 90.69 & 97.13 & 99.89 & 100 \\
Corn senesced green weeds & 83.12 & 84.61 & 95.25 & 99.17 & 99.89 \\
Lettuce romaine 4 wk & 10.19 & 81.50 & 83.58 & 99.77 & 99.81 \\
Lettuce romaine 5 wk & 90.26 & 92.17 & 91.47 & 99.77 & 100 \\
Lettuce romaine 6 wk & 94.90 & 97.57 & 92.73 & 98.79 & 99.90 \\
Lettuce romaine 7 wk & 78.17 & 99.38 & 89.81 & 92.72 & 99.74 \\
Vinyard untrained & 41.36 & 54.32 & 56.23 & 67.25 & 99.76 \\
Vinyard vertical trellis & 87.71 & 98.40 & 98.53 & 98.77 & 99.76 \\
Overall Accuracy & 69.13 & 79.02 & 92.16 & 92.19 & 99.87 \\
\hline
\end{tabular}


of all compared methods (PCA, LLE, and ISS-WME) in Tables 4-9 are copied from [13]. The best results are highlighted in bold font. Our proposed method in this paper improves upon existing methods for at least $10 \%$ in classification rates for almost all cases in our experiments.

\section{Conclusions}

In this paper, we have proposed a novel method for HSI classification by means of PCA and SVM. We perform PCA on existing HSI data cube to reduce the dimensionality of the data cube. We spatially convolve the PCA output cube with three different $2 \mathrm{D}$ filters. We use SVM to classify every pixel to one of the known classes for the three convolved output cubes. We perform fusion on the three maps from SVM to generate the final classification map. Our new method improves upon several existing methods in term of classification rate significantly, and it is relatively fast as well because it only performs simple operations on the HSI data cubes.

\section{REFERENCES}

[1] Y. Guo, X. Yin, X. Zhao, D. Yang and Y. Bai, "Hyperspectral Image Classification with SVM and Guided Filter", EURASIP Journal on Wireless Communications and Networking, Article number: 56, 2019.

[2] F. Zhou, R. Hang, Q. Liu and X. Yuan, "Hyperspectral Image Classification using Spectral-Spatial LSTMs", Neurocomputing, vol. 328, pp. 39-47, 2019.

[3] Y. Chen, N. M. Nasrabadi and T. D. Tran, "Hyperspectral Image Classification via Kernel Sparse Representation", IEEE Transactions on Geoscience and Remote Sensing, vol. 51, no. 1, pp. 217-231, 2013.

[4] F. Melgani and L. Bruzzone, "Classification of Hyperspectral Remote Sensing Images with Support Vector Machines", IEEE Transactions on Geoscience and Remote Sensing, vol. 42, no. 8, pp. 1778-1790, 2004.

[5] M. Fauvel, J. Chanussot and J. A. Benediktsson, "A Spatial-Spectral Kernel-Based Approach for the Classification of Remote-Sensing Images", Pattern Recognition, vol. 45, no. 1, pp. 381-392, 2012.

[6] G. Camps-Valls and L. Bruzzone, "Kernel-Based Methods for Hyper-Spectral Image Classification", IEEE Transactions on Geoscience and Remote Sensing, vol. 43, no. 6, pp. 1351-1362, 2005.

[7] J. Li, P. R. Marpu, A. Plaza, J. M. Bioucas-Dias and J. A. Benediktsson, "Generalized Composite Kernel Framework for Hyper-Spectral Image Classification", IEEE Transactions on Geoscience and Remote Sensing, vol. 51, no. 9, pp. 4816-4829, 2013.
[8] Y. Chen, N. M. Nasrabadi and T. D. Tran, "Hyperspectral Image Classification using Dictionary-Based Sparse Representation", IEEE Transactions on Geoscience and Remote Sensing, vol. 49, no. 10, pp. 3973-3985, 2011.

[9] J. Li, J. M. Bioucas-Dias and A. Plaza, "Spectral-Spatial Classification of Hyperspectral Data using Loopy Belief Propagation and Active Learning", IEEE Transactions on Geoscience and Remote Sensing, vol. 51, no. 2, pp. 844-856, 2013.

[10] X. D. Kang, S. Li and J. A. Benediktsson, "Spectral-spatial HyperSpectral Image Classification with Edge-Preserving Filtering", IEEE Transactions on Geoscience and Remote Sensing, vol. 52, no. 5, pp. 2666-2677, 2014.

[11] G. Cheng, F. Zhu, S. Xiang, Y. Wang and X. Pan, "Semisupervised Hyperspectral Image Classification via Discriminant Analysis and Robust Regression", IEEE Journal of Selected Topics in Applied Earth Observations and Remote Sensing, vol. 9, no. 2, pp. 595-608, 2016.

12] Y. Chen, Z. Lin, X. Zhao, G. Wang and Y. Gu, "Deep Learning-Based Classification of Hyperspectral Data", IEEE Journal of Selected Topics in Applied Earth Observations and Remote Sensing, vol. 7, no. 6, pp. 2094-2107, 2014.

[13] H. Liu, K. Xia, T. Li, J. Ma and E. Owoola, "Dimensionality Reduction of Hyperspectral Images Based on Improved Spatial-Spectral Weight Manifold Embedding",Sensors 2020, 20, 4413; doi10.3390/s20164413.

[14] I. T. Jolliffe, Principal Component Analysis, second edition (Springer), 2002.

[15] C. Cortes and V. N. Vapnik, "Support-Vector Networks", Machine Learning, vol. 20, no. 3, pp. 273-297, 1995.

[16] S. Damelin and W. Miller, The mathematics of signal processing, Cambridge University Press, ISBN 978-1107601048, 2011.

[17] C. C. Chang and C. J. Lin. "LIBSVM: a Library for Support Vector Machines", ACM Transactions on Intelligent Systems and Technology, vol. 2, no. 27, pp. 1-27, 2011.

[18] S. Roweis and L. Saul, "Nonlinear Dimensionality Reduction by Locally Linear Embedding", Science, vol. 290, no. 5500, pp. 2323-2326, 2000.

Received 1 November 2020

Guang Yi Chen holds a BSc in Applied Mathematics, an MSc in Computing Mathematics, an MSc in Computer Science, and a PhD in Computer Science. During his graduate and postdoctoral studies in Canada, he was awarded many prestigious fellowships. He has published over fifty scientific journal papers in his fields and holds two granted US patents in image processing. He is currently affiliated to the Department of Computer Science and Software Engineering, Concordia University, Montreal, Quebec, Canada. His research interests include pattern recognition, image processing, machine learning, artificial intelligence, remote sensing, and scientific computing. 